Departamento de Anatomía Patológica. Facultad de Medicina.

Universidad de La Frontera. Temuco, Chile.

aresidente Especialidad Anatomía Patológica.

No hubo apoyo financiero de ninguna índole.

Recibido el 1 de octubre de 2013, aceptado el 16 de abril de 2014 .

Correspondencia a:

Dr. Enrique Bellolio Manuel Montt 112 Temuco, Chile.

Código Postal: 4781176 ebellolio@gmail.com

\section{Enfermedad de Castleman, un diagnóstico diferencial que no se debe olvidar en los tumores de mediastino. Caso clínico}

\author{
ENRIQUE BELLOLIO J. ${ }^{1}$, OSCAR TAPIA ${ }^{1}, \mathrm{M}^{\mathrm{a}}$ JOSÉ IRIARTE C. ${ }^{\mathrm{a}}$
}

Castleman's disease is an uncommon lymphoproliferative disorder which can be unicentric or multicentric. Hialine vascular variant is the most common pathologic form, which is usually unicentric and presenting as mediastinal tumors. We report a 31-year-old female with a history of retrosternal pain. A chest CAT scan showed a tumor in the posterior mediastinum. The patient was operated and the tumor excised. The pathology report showed a Castleman's disease. No other tumors were found in the patient, who had a favorable evolution.

(Rev Med Chile 2014; 142: 782-785)

Key words: Giant Lymph Node hyperplasia; Mediastinum; Tomography scanners, $X$-ray computed.

L os tumores de mediastino generalmente se presentan como un hallazgo incidental en una radiografía de tórax o tomografía axial computada, solicitado por otra patología subyacente del paciente en estudio.

Las lesiones tumorales malginas del mediastino son poco frecuentes, siendo las lesiones benignas un desafío diagnóstico para los radiólogos y patólogos. En general estas lesiones tienen patrones imagenológicos que sugieren ciertas entidades diagnósticas, pero cuando no es posible tener una certeza, los cirujanos de tórax se apoyan en el diagnóstico de la biopsia intraoperatoria para definir conductas terapéuticas.

\section{Caso clínico}

Presentamos el caso de paciente de sexo femenino de 31 años sin antecedentes mórbidos de importancia, con historia de dolor retroesternal de 3 meses de evolución con examen físico anodino y exámenes de laboratorio dentro de rangos nor- males. Se solicitó radiografía de tórax que mostró lesión en mediastino medio-posterior derecho. Se completó estudio con tomografía axial computarizada (TAC) que mostró tumor de mediastino posterior, sugerente de paraganglioma.

Se realizó resección quirúrgica de la lesión con biopsia intraoperatoria que evidencia enfermedad de Castleman (EC) de tipo hialino vascular; posterior a lo cual la paciente fue estudiada para descartar compromiso sistémico, presentando evolución favorable.

El estudio macroscópico demostró tumor de mediastino, ovalado de $29 \mathrm{~g}$, de $5 \mathrm{~cm}$ por $4,5 \mathrm{~cm}$ por $2 \mathrm{~cm}$, de superficie pardo rosada en partes violácea de aspecto hemático, al corte se vió superficie pardo rosada, finamente granular homogénea (Figura 1).

El examen microscópico reveló hiperplasia angiofolicular linfoide característica con disposición de linfocitos en "tela de cebolla" alrededor (Figuras 2 y 3 ). El estudio con inmunohistoquímica mostró patrón reactivo con $\mathrm{Bcl}-2(-)$ (Figura 4), células reactivas $\mathrm{CD} 3(\mathrm{sp} 7)(+), \mathrm{CD} 10(-), \mathrm{CD} 20(++)$, 
Enfermedad de Castleman - E. Bellolio et al

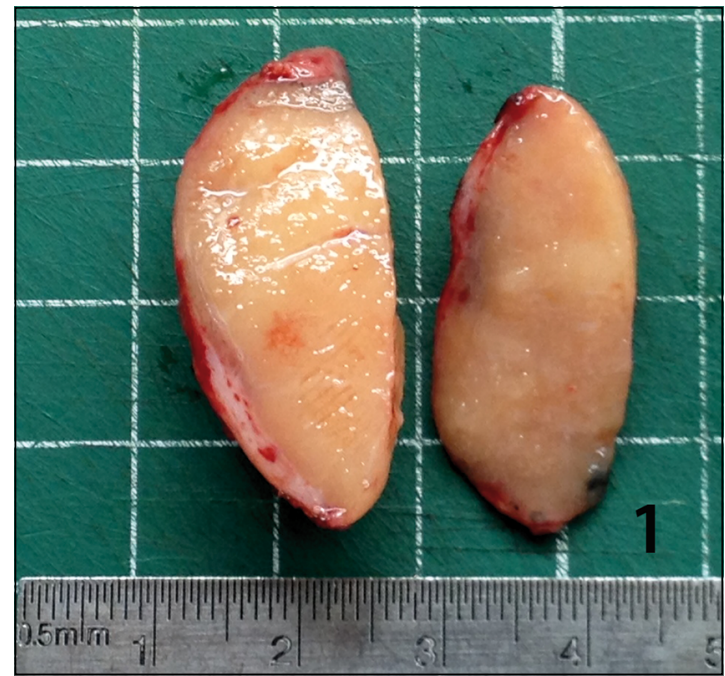

Figura 1.

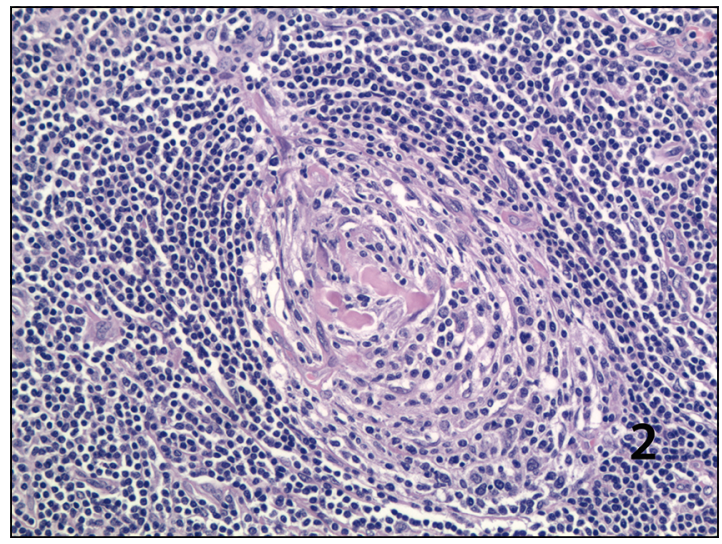

Figura 2.

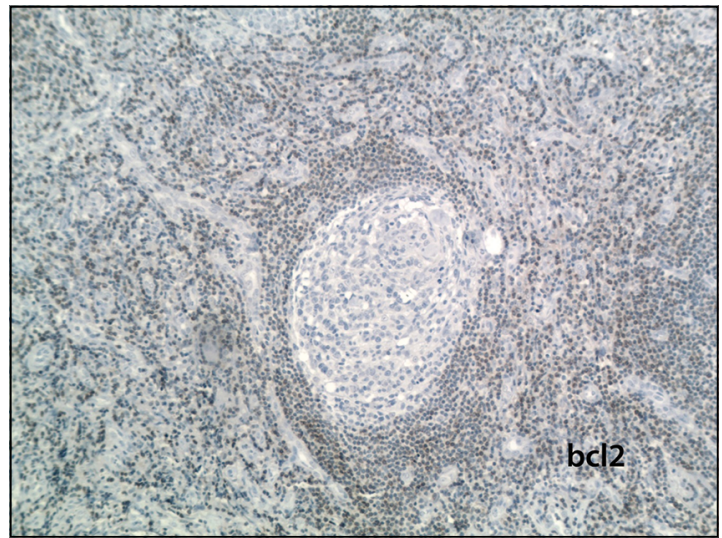

Figura 4. células centrogerminales CD43 (+) (Figura 5), células reactivas y CD30 (-).

La enfermedad de Castleman, conocida también como hiperplasia nodular gigante no-clonal, hiperplasia angiofolicular linfoide, linforeticuloma folicular, linfoma gigante benigno, hamartomalinfoide, es una entidad infrecuente, de incidencia desconocida, enmarcada dentro de los trastornos linfoproliferativos con etiopatogenia aún no del todo dilucidada ${ }^{1,2,6,8,9}$.

\section{Discusión}

Existen tres variantes histológicas: hialinovascular, que ocurre en $80-90 \%$ de los casos, habitualmente es asintomática, se presenta generalmente en casos localizados o afectando a un grupos de

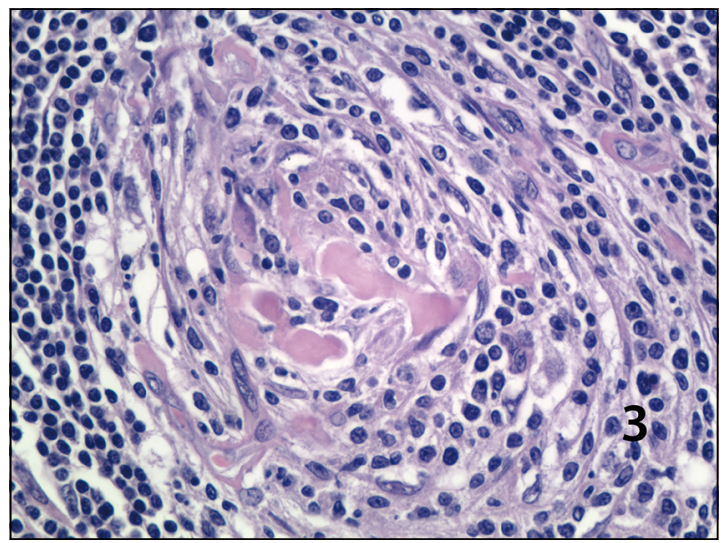

Figura 3.

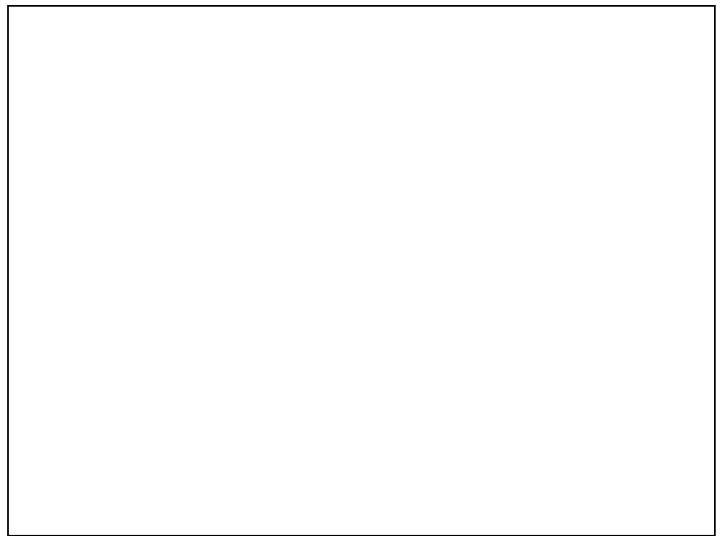

Figura 5. 
linfonodos principalmente en mediastino, $60 \%$; cuello, 14\%; abdomen y retroperitoneo $11 \%$, predomina en la cuarta década de la vida. Plasmocelular ocurre en 10\%, se presenta clínicamente de forma multicéntrica o en varios grupos de linfonodos y generalmente acompañados de síntomas sistémicos como compromiso del estado general. En general esta última con un curso más agresivo. La tercera variante es la mixta, una mezcla de las anteriores ${ }^{1-8}$.

En la presentación mediastínica, la evaluación radiológica con TAC muestra una masa mediastinal o hiliar, homogéneamente reforzada después de contraste parecido a un timoma o cáncer de pulmón. En la TAC se describen tres patrones diferentes del tipo hialino-vascular: masa solitaria no invasiva $(50 \%)$, masa infiltrante con linfadenopatía asociada $(40 \%)$ o sin linfadenopatía masiva. En ultrasonografía Doppler se ve imagen con hipervascularidad periférica ${ }^{8,14}$.

A pesar de los avances en el estudio de esta enfermedad, su etiopatogenia es confusa y probablemente diversa, siendo recientemente considerada la forma generalizada como un entidad clínica separada ${ }^{5,6}$. En general se ha aceptado que corresponde a una estimulación antigénica crónica de probable etiología viral o una alteración en la regulación de los factores de crecimiento que conduce a una proliferación anómala de células plasmáticas. Se ha visto en tejidos afectados por esta patología elevados índices de IL-6, lo que se asocia con proliferación de células $\mathrm{B}$ y diferenciación a células plasmáticas, la que también podría provocar muchas de las manifestaciones patológicas ${ }^{6,7}$.

Se han descrito asociaciones a otras patologías como síndrome de POEMS, depósitos de amiloide, plasmocitoma, linfoma folicular, linfoma de Hodgkin, sarcoma de Kaposi y microangiopatíatrombótica renal ${ }^{5,8}$.

El diagnóstico diferencial debe incluir patologías que se presentan con grandes masas mediastínicas como linfomas de Hodgkin, timomas, teratomas y ciertas enfermedades reumatológicas que pueden causar adenomegalias ${ }^{7}$.

El diagnóstico de confirmación es histológico, requiriéndose la resección completa del ganglio afectado. La biopsia por aspiración con aguja fina no es útil para el diagnóstico ${ }^{2,3,6}$.

En cuanto a la histología se describe en la forma hialinovascular como hiperplasia o proliferación folicular anómala con importante vascularización interfolicular y zona del manto gruesa. Cada folículo puede contener varios centros germinales, la mayoría de los folículos se encuentran rodeados de una densa red capilar que penetra hasta llegar al centro germinal (que se puede demostrar con tinción P.A.S.) rodeados de un grueso armazón de colágeno, que se observa en tinción de reticulina, entre ellos pueden encontrarse linfocitos pequeños dispersos o agrupados, células plasmáticas y monocitos plasmacitoides, también puede tener inmunoblastos, eosinófilos y neutrófilos. Desaparecen las estructuras sinusales. Algunos de los centros foliculares están rodeados por una estrecha capa de pequeños linfocitos en anillos concéntricos que confieren una típica imagen "en bulbo de cebolla" y también presentan degeneración hialina. Los centros germinales de esta entidad son hipocelulares y están constituidos mayoritariamente por células dendríticas, células endoteliales de la red capilar, células reticulares y pocas células B con pocas atipias.

El diagnóstico se puede complicar cuando hay pocos o muchos folículos hialino vasculares lo que podría parecer un ganglio linfático reactivo, como lo que se puede observar en las últimas etapas de la infección por HIV. En estos casos puede encontrarse sinusoides residuales con algunas células plasmáticas y carecer de la red vascular interfollicular.

Los estudios inmunohistoloquímicos muestran un patrón policlonal de linfocitos $\mathrm{B}, \mathrm{T}$ y de células plasmáticas (CD3 y CD20 positivos). Este patrón histológico del centro germinal no es específico de EC y puede encontrarse en nódulos linfáticos de pacientes con SIDA y en asociación con linfadenopatías angioinmunoblásticas.

En la variante histológica plasmocelular, la arquitectura del nódulo linfático está conservada, se caracteriza por la presencia de abundantes células plasmáticas en el espacio interfolicular con menos vascularización y los folículos linfoides de mayor tamaño con sus centros germinales más prominentes. En la mayoría de los casos las células plasmáticas tienen expresión de la cadena ligera de inmunoglobulinas polipeptípicas y un tercio muestra restricción de la cadena ligera (lambda); también se encuentra expresión policlonal de células plasmáticas.

El principal diagnóstico diferencial histológico es con adenopatías reactivas con marcada plasmo- 
citosis, como se ve en la enfermedad reumatoide y sífilis. En ellos la estructura subyacente del seno es por lo general más evidentes y el infiltrado interfolicular plasmocítico menos uniforme $\mathrm{1}^{1,2,11-13,15}$.

Puede progresar a una fase "burn-out" con abundantes centros germinales vascular hialino $y$ se puede interpretar como mixto.

El tratamiento de la forma localizada es la resección quirúrgica completa del ganglio linfático afectado, tanto en el tipo hialino vascular como el tipo plasmocelular; con una curación de casi $100 \%$ de los casos y con excepcionales recurrencias. En la forma multicéntrica, es difícil hablar de tratamiento ideal, dado el escaso número de pacientes de las series publicadas y la amplia variabilidad de los resultados descritos en la literatura. Está recomendado el screening en los casos diagnosticados para confirmar enfermedad localizada o sistémica $^{10,11}$.

El seguimiento a largo plazo y la vigilancia periódica aún no están definidos para ninguna de las formas clínicas ${ }^{1,2}$.

\section{Referencias}

1. Coca PI, Ortega JM, Fernández RE, Gavilán CJ, Bermúdez RF. Enfermedad de Castleman localizada: descripción de un caso y revisión de la literatura. An Med Interna Madrid 2003; 20 (10): 534-6.

2. Sánchez de Toledo SJ, Fábrega SJ, Marhuenda IC, Lucaya LX, Torán FN, Gros SL, et al. Enfermedad de Castleman. An Pediatr (Barc) 2005; 63 (1): 68-71.

3. Castleman disease, American Cancer Society, disponible en http://www.cancer.org/acs/groups/cid/documents/ webcontent/003093-pdf.pdf (revisado por última vez el 19 de junio de 2013).
4. Sepúlveda M, Contreras E, Martínez N, Enfermedad de Castleman, Descripción de un caso clínico. Rev Venez Oncol 2008; 20 (2): 104-7.

5. Camacho R, Millán F, Scher P, Castrillón C, Panzuto O. Enfermedad de Castleman: presentación de un caso y revisión bibliográfica. Rev de Cir Infantil 2004; 14: 1-4.

6. Roca B. Castleman's Disease. A Review. AIDS 2009; 11: 3-7.

7. Hountis P, Dedeilias P, Douzinas P. The management of Castleman's disease of the mediastinum: a case report. Cases Journal 2008; 1: 330.

8. Paci M, Valli R, Tenconi S, Sgarbi G. Mediastinal Castleman's disease mimicking thoracic paravertebral schwannoma, Paci y cols. Interactive CardioVascular and Thoracic Surgery 2011; 13: 346-7.

9. Sarrot-Reynauld F. Castleman's Disease, 2001, Orphanet encyclopedia, disponible en www.orpha.net/patho/GB/ uk-castleman.pdf, revisado por última vez el 19 de junio de 2013.

10. Regal M, Aljehani Y, Bousbait H. Primary mediastinal Castleman's disease. Rare Tumors 2010; 2: e11.

11. Rodríguez CJ, Fernández MC, López PC, Muñoz HA, Cámara PM. Enfermedad de Castleman: un caso de presentación atípica. An Pediatr (Barc) 2009; 70 (1): 61-4.

12. Dennis H. Wright. Diagnostic Lymph Node Pathology. Oxford University Press Inc. 2006; 20-7.

13. Ioachim H. Castleman lymphadenopathy. Lymph Node Pathology. Lippincott Williams \& Wilkins 2002; 227-37.

14. Jin KY, Wook CB, Seung SJ, Ok CK. Mediastinal Castleman disease: heterogeneous enhancement withfilling-in pattern on dynamic CT and MRI, European Journal of Radiology Extra 2004; 52: 103-5.

15. Sousa V, Carvalho L. Hiperplasia Linfóide Angiofolicular-Doença de castleman-do mediastino: Apresentação de Um Caso Clínico. Revista Portuguesa de Pneumología, 2006; xii. 